\title{
Selectivity of Candida rugosa Lipase immobilized onto layered double hydroxides as catalyst in synthesis of fatty acid esters
}

\begin{abstract}
The enzymatic selectivity of Lipase from Candida rugosa immobilized onto a calcined layered double hydroxide (CLDHs-CRL) towards the chain-length of fatty acids and alcohols in the synthesis of fatty acid esters was investigated. The results showed that CMAN-CRL catalyzed the esterification process with fatty acids of medium chain lengths (C10-C14) effectively while, CNAN-CRL and CZAN-CRL exhibited high percentage conversion in fatty acids with carbon chain lengths of $\mathrm{C} 8-\mathrm{C} 12$ and $\mathrm{C} 10-\mathrm{C} 18$, respectively. In the alcohol selectivity study, CMAN-CRL showed high selectivity toward alcohols with carbon chain lengths of C4, C6 and C10. On the other hand, both CNAN-CRL and CZAN-CRL exhibited rather low selectivity towards longer carbon chain length of alcohols.
\end{abstract}

Keyword: Candida rugosa lipase; Immobilized; Layered double hydroxides; Fatty acid esters 